Ann. Biol. anim. Bioch. Biophys., r976, 16 (I), I2I-128.

\title{
EFFETS DU MAGNÉSIUM SUR DES MEMBRANES LIQUIDES ET BIMOLÉCULAIRES PHOSPHOLIPIDIQUES. L'ION MAGNÉSIUM COMPÉTITEUR DES CATIONS MONOVALENTS
}

\author{
M. BARA (1) \\ Laboratoire de Physiologie générale, \\ Universite Paris VI, \\ 9, quai Saint-Bernard, \\ Paris 75005

\section{RÉSUMÉ}

La structure des membranes cellulaires est encore mal connue : elles sont formées d'entités lipidiques et protéiniques, et certains paramètres physiques (nature des espèces ioniques par exemple) agissent sur l'orientation des molécules lipidiques et sur les protéines. En utilisant des membranes artificielles de type "liquide " (phospholipides dissous dans un solvant organique à faible constante diélectrique et imprégnant un filtre millipore en téflon), ou des membranes bimoléculaires phospholipidiques, nous avons voulu mettre en évidence le rôle des cations divalents et en particulier du $\mathrm{Mg}^{++}$. Les mesures effectuées sont des mesures de résistance et des mesures de potentiel de membrane (à l'aide d'un électromètre Keithley 6roB).

Le magnésium et les autres cations divalents agissent sur la stabilité structurale de la membrane : cette action se manifeste par une augmentation de la résistance des membranes à charge résultante positive (Céphaline et Lécithine), et une diminution de celle ayant une charge résultante négative (lipides totaux de cerveau de bœuf) ; en outre, on enregistre une action sur la différence de potentiel membranaire (beaucoup plus nette avec les lipides totaux).

L'action de $\mathrm{Mg}^{++}$est explicable par application de la théorie de la double couche de Gour et Chapman ; $\mathrm{Mg}^{++}$agit par effet d'écran ; $\mathrm{Mg}^{++}$est adsorbé spécifiquement sur les groupes polaires de la membrane, et ce point est confirmé par les courbes $I / g=f\left(\mathrm{Mg}^{++}\right)$qui montrent que $\mathrm{Mg}^{++}$est un compétiteur des cations monovalents au niveau des mêmes sites de la membrane.

\section{INTRODUCTION}

Les neurophysiologistes, depuis les travaux de HoDGkIN et HuXLEY (I952), se sont particulièrement intéressés à l'étude des mouvements des différents cations

(1) Laboratoire de Biologie de la Reproduction, Université Paris VI, Tour 32 et Bât. G., 4 place Jussieu, 75005 Paris. 
mono et divalents, qui accompagnent les phénomènes électriques de l'excitabilité nerveuse.

Les nombreuses questions qui restent posées, commenceraient à avoir un début de réponse, si on connaissait mieux la structure des membranes cellulaires. En résumé, à l'heure actuelle, nous sommes en présence de deux théories concernant celle-ci :

a) Gor'ter et Grendel, (I925), puis Danielili et Davson (I935), émirent l'hypothèse d'une double couche de molécules lipidiques réunies par leurs extrémités hydrophobes et intercalées entre deux feuillets protéiniques qui formeraient les surfaces interne et externe de la membrane. Ce modèle rigide, rend difficilement compte des différences de perméabilité interionique ; aussi STEIN et DANIELII (I956) envisagèrent l'existence de pores permanents bordés de protéines.

b) Cette théorie fut controversée à partir de I963 par SJösTRAND, qui montre la présence de micelles formés de lipoprotéines; LUCY (I964) émit 1'hypothèse de la présence de sous-unités globulaires lipidiques enrobées de protéines ou de glycoprotéines qui laisseraient entre elles des pores de $4 \AA$ de diamètre, lieu de perméation.

Bien d'autres modèles virent le jour, et nous citerons pour mémoire ceux de : Benson (i966), Greein et Perdue (I966), Lenard et Singer (I966).

Ces hypothèses reviennent à poser les questions suivantes :

- le rôle des protéines dans la sélectivité de la membrane;

- le rôle structurale des lipides;

- l'effet de paramètres physiques (concentration des ions, nature des ions environnant les membranes cellulaires...) sur l'organisation des molécules lipidiques ; point qui nous a semblé particulièrement intéressant, avec l'étude du magnésium en particulier.

Pour répondre à ces questions, nous avons envisagé l'étude des modèles de membranes qui sont simples et formés d'éléments connus (phospholipides dissous dans un solvant organique).

\section{MATÉRIEL ET MÉTHODES}

\section{A. - Membranes artificielles de type "liquide"}

\section{r. Caractéristiques.}

Ce sont des membranes échangeuses d'ions utilisées pour étudier dans un milieu totalement anhydre, les interactions entre les phospholipides principaux existant dans les membranes cellulaires et les cations mono et divalents (en particulier le magnésium, ayant des effets souvent antagonistes du calcium).

\section{Formation et montage expérimental (fig. I).}

La membrane est formée par simple imprégnation, pendant $4^{8}$ heures d'un filtre millipore en téflon (type Mitex TM, épaisseur I $50 \mu$, porosité $60 \mathrm{p}$. Ioo, diamètre des pores $5,0 \mu$ : ce matériel est peu mouillable par l'eau et est essentiellement lipophile), trempé dans une solution de : heptane-chloroforme-méthanol : 7:2: I et de phospholipides : 2 p. Ioo; 1'ensemble formant un système hétérogène comportant une matrice hydrophobe et des pores aqueux, ayant une densité 
élevée de sites. Le filtre est inséré entre deux chambres de plexiglas contenant les solutions aqueuses à $36^{\circ} \mathrm{C}$ et $\mathrm{pH} 7,4$. Les mesures électriques (potentiel, résistance) ont été réalisées à l'aide d'un électromètre Keithley 6roB.

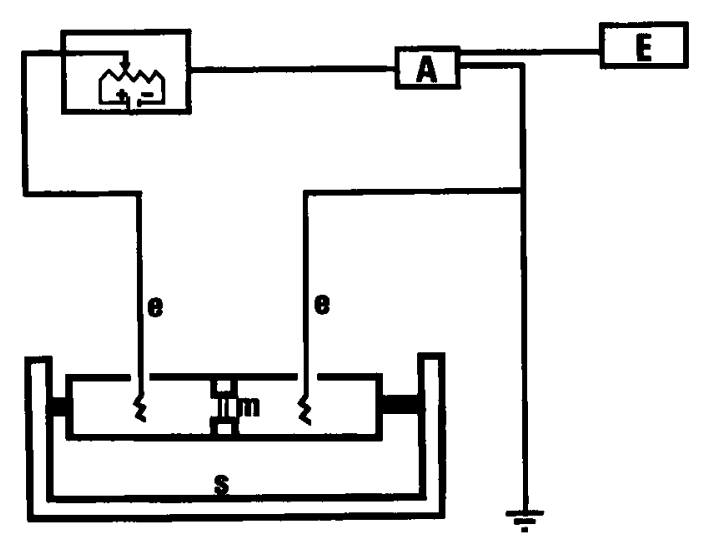

FIG. I. - Schéma de montage pour l'étude des membranes "liquides"

$\mathrm{m}$ : membrane : support en téfion

$\mathrm{s}$ : support métallique

$\mathrm{e}$ : électrodes en chlorure d'argent

A : Keithley 6 IoB (impédance d'entrée ro ${ }^{14} \mathrm{ohms}$ )

fonctionnant en ampèremètre

$\mathrm{E}$ : enregistreur

B. - Membranes artificielles bimoléculaires phospholipidiques

\section{Caractéristiques.}

Ce sont des doubles couches mono-moléculaires formant un système fluide (KRASNE, 1971), avec une perméabilité ionique faible; mais différentes des membranes liquides par : l'épaisseur (6o $\AA$ contre plusieurs microns) ; l'organisation des têtes polaires.

Ce type de membrane (de structure proche de celle des membranes cellulaires), a permis de mettre en évidence le rôle de la nature des espèces ioniques.

\section{Formation et montage expérimental.}

Les membranes bimoléculaires sont formées soit de l'ensemble des lipides du cerveau de bœuf, soit de phosphatidyl-éthanolamine, avec de l'a-tocophérol, du cholestérol, préalablement dissous dans un mélange chloroforme-méthanol $2:$ I.

Le dispositif expérimental choisi est très simple, et consiste en deux chambres qui communiquent par un petit trou $\left(0,75 \mathrm{~mm}^{2}\right)$, sur lequel la membrane se forme. Nous avons employé l'appareillage de MUELLER et coll. (1962) : la chambre interne est faite d'une petite coupe en téflon, la chambre externe est en plexiglas. Au niveau du trou, la membrane se forme par dissolution du solvant dans la phase aqueuse (à $3^{\circ} \mathrm{C}$ et $\mathrm{pH} 7,4$ ) et par " drainage " des molécules lipidiques, pour atteindre l'état stable : l'état bimoléculaire en équilibre avec l'anneau périphérique. $6 \mathrm{roB}$.

Les mesures de résistance, de potentiel, ont été réalisées à l'aide d'un électromètre Keithley

\section{RÉSULTATS}

Nous avons étudié l'action des cations divalents et du magnésium en particulier, sur la résistance et sur la différence de potentiel membranaire. 


\section{A. - Action sur la résistance}

Les cations divalents, sous forme de chlorures, $\mathrm{pH} 7,4$, sont ajoutés d'un seul côté de la membrane.

I. Membranes "liquides ": (Céphaline 2 p. Ioo).

Nous avons observé :

- la résistance de la membrane augmente légèrement lorsque le magnésium, à la concentration de Io $\mathrm{mM}$, traverse la membrane :

$$
\begin{aligned}
& R \text { sans divalent }: 6,0 \pm 0, I \cdot \mathrm{IO}^{4} \mathrm{ohms} \cdot \mathrm{cm}^{-2} \\
& R \text { avec divalent }: 7,6 \pm 0, \mathrm{I} \cdot \mathrm{IO}^{4} \mathrm{ohms} \cdot \mathrm{cm}^{-2}
\end{aligned}
$$

- la séquence d'action des cations divalents sur la résistance de la membrane est comparable à la séquence $\mathrm{V}$ d'Eisenman :

$$
\mathrm{Ca}^{++}>\mathrm{Mg}^{++}>\mathrm{Ba}^{++}>\mathrm{Sr}^{++}
$$

\section{Membranes bimoléculaires.}

a) Lipides totaux (fig. 2).

Nous avons observé :

- la résistance de la membrane diminue légèrement jusqu'à $5 \mathrm{mM}$ de magnésium, puis fortement au-dessus de Io-I2 mM : il y a rupture de la membrane.

- la séquence d'action des cations divalents est la suivante :

$$
\mathrm{Ba}^{++}>\mathrm{Ni}^{++}>\mathrm{Mn}^{++}>\mathrm{Ca}^{++}>\mathrm{Mg}^{++}>\mathrm{Sr}^{++}
$$

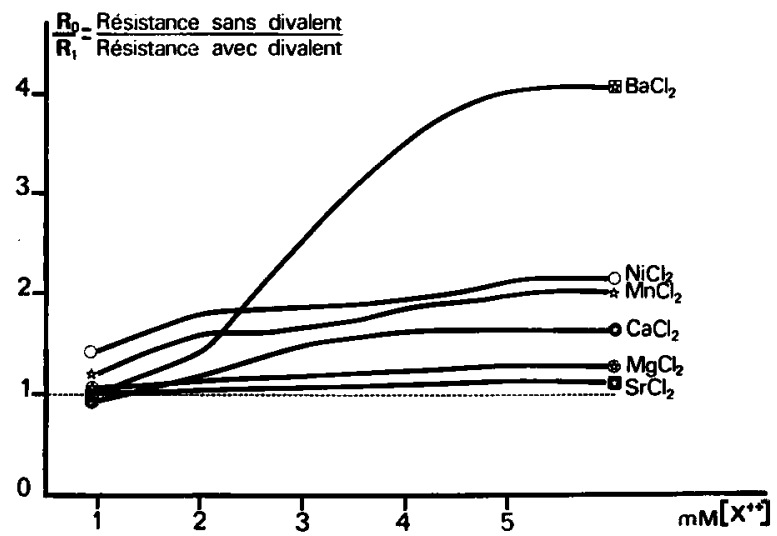

FIG. 2. - Membranes bimoléculaires formées de lipides totaux : action des cations divalents sur la résistance de la membrane (la figure montre l'action de $\mathrm{Mg}^{++}$jusqu'à $6 \mathrm{mM}$ ). Pour tous les cations, la résistance diminue. (Chaque point correspond à la moyenne de résultats obtenus sur un ensemble de 25 membranes différentes; l'écart-type calculé étant très faible).

\section{b) Lécithine.}

Nous avons observé :

- la résistance de la membrane augmente légèrement en présence de $\mathrm{Mg}^{++}$ et des cations monovalents : $\mathrm{K}^{+}, \mathrm{Na}^{+}, \mathrm{Li}^{+}, \mathrm{Cs}^{+}, \mathrm{Rb}^{+}$. 


\section{Comparaison.}

Quel que soit le modèle employé, en présence de $\mathrm{Mg}^{++}$, la résistance de la membrane augmente, si celle-ci est constituée de phospholipides ayant une charge résultante positive (céphaline, lécithine); la résistance diminue en présence de phospholipides à charge résultante négative (lipides totaux). Dans ce dernier cas, quand la concentration en $\mathrm{Mg}^{++}$augmente, la résistance décroît fortement, car le cation divalent pénètre dans la membrane et détruit le réseau organisé des têtes polaires.

\section{B. - Action sur la différence de potentiel membranaire}

Les cations divalents, sous forme de chlorures, $\mathrm{pH} 7,4$, sont ajoutés d'un seul côté de la membrane.

I. Membranes "liquides": (Céphaline 2 p. I00).

L'addition de $\mathrm{Mg}^{++}$provoque une augmentation linéaire de la différence de potentiel en fonction de la concentration en cation divalent : $5 \mathrm{mV}$ pour Io $\mathrm{mM}$.

2. Membranes bimoléculaires.

a) Lipides totaux (fig. 3).

L'addition de $\mathrm{Mg}^{++}$provoque une augmentation linéaire de la différence de potentiel : I2 à $I_{5} \mathrm{mV}$ pour Io $\mathrm{mM}$.

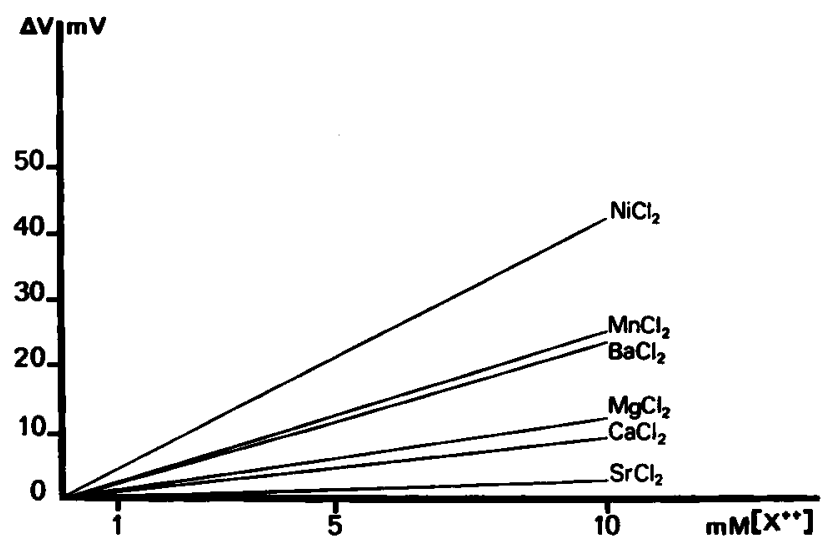

FıG. 3. - Membranes bimoléculaires formées de lipides totaux : action des cations divalents sur la différence de potentiel ; ceux-ci sont ajoutés d'un seul côté de la membrane. Cette courbe montre que $\mathrm{Mg}^{++}, \mathrm{Ca}^{++}$, $\mathrm{S} \mathbf{r}^{++}$, agissent par effet d'écran; $\mathrm{Ni}^{++}, \mathrm{Mn}^{++}, \mathrm{Ba}^{++}$, par liaisons fortes.

\section{b) Lécithine.}

$\mathrm{Mg}^{++}$provoque une augmentation linéaire de la différence de potentiel de : 2 à $5 \mathrm{mV}$ pour Io $\mathrm{mM}$.

\section{Comparaison.}

L'addition de $\mathrm{Mg}^{++}$a une action beaucoup plus prononcée avec des membranes à charge résultante négative (lipides totaux) qu'avec des phospholipides ayant une 
charge résultante positive (dans les molécules de lécithine et de céphaline, le nombre de groupes $\mathrm{PO}_{3}^{-}$et $\mathrm{NH}_{3}^{+}$est identique, mais on peut admettre que certains groupes négatifs sont masqués et les groupes positifs sont en nombre supérieur; la charge résultante étant alors positive.

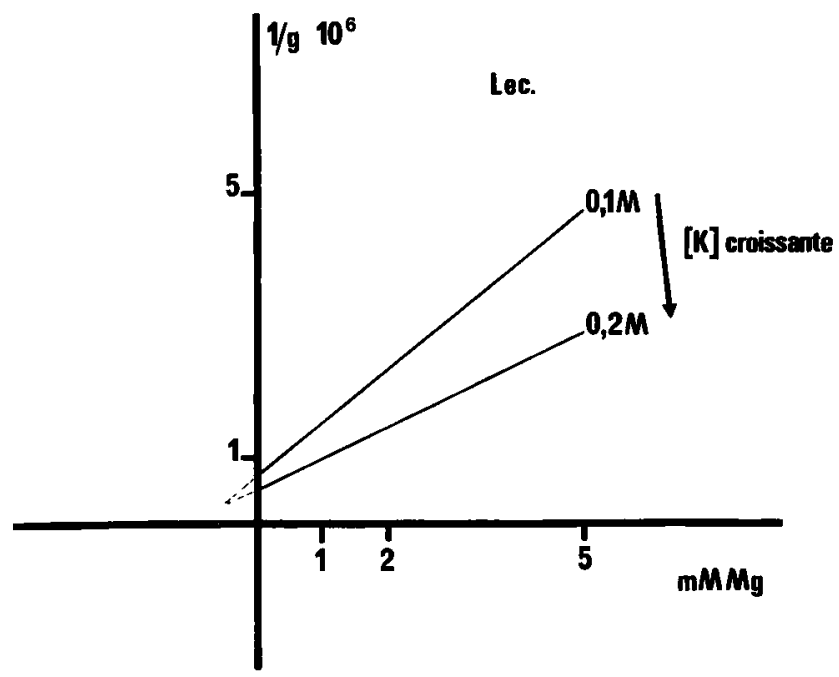

Fig. 4. - Membranes bimoléculaires formées de Lécithine: $I / g=f\left(\mathrm{Mg}^{++}\right):$pour une concentration constante de $\mathrm{K}^{+}, \mathrm{I} / \mathrm{g}$ varie de façon linéaire en fonction de l'augmentation de la concentration en ions $\mathrm{Mg}^{++}$, celui-ci pouvant être alors assimilé à un inhibiteur. Nous sommes en présence d'une inhibition compétitive (représentation des cinétiques enzymatiques de Dixon). L'inhibition est levée par un excès de $\mathrm{K}^{+} ; \mathrm{Mg}^{++}$et $\mathrm{K}^{+}$se fixent au niveau des mêmes sites sur le phospholipide.

\section{DISCUSSION}

I. L'étude de l'action de $\mathrm{Mg}^{++}$sur des membranes artificielles, nous a permis de montrer l'influence des interactions cations divalents-phospholipides sur la stabilité structurale de la membrane (influence manifestée par une action sur la résistance et sur la différence de potentiel des membranes).

(I) L'action sur le potentiel est explicable par la théorie de la double couche de Gouy et Chapman (McDonald et al., I972), qui montre :

a) la nécessité de la présence de charges négatives ou positives pour avoir un effet ;

b) la distinction entre une action des ions divalents par effet d'écran, c'est-à-dire, les charges positives fixées à l'interface repoussant les cations ; ou fixation par liaisons électrostatiques fermes ou covalentes sur les groupes polaires (Gri,BERT et EHRENSTEIN, I969).

- La mesure de potentiel réalisée au cours de nos expériences est une mesure directe, contrairement à celle faite par MUELLER et al. (I962), McLAUGHIIN et al. (I97I), qui réalisent des mesures indirectes; mais McDonald et BANGHAM (I972) font une mesure directe rendue possible, car les membranes sont très peu conductrices, et les fuites, très faibles. 
- Les potentiels enregistrés sont des potentiels d'interface et non pas des potentiels de diffusion. En effet, l'observation des constantes de temps est un bon argument dans ce sens : pour un potentiel de diffusion, le temps d'établissement du potentiel ne peut excéder le temps de charge $\mathrm{RC}$ d'un circuit ayant la constante de temps de la membrane; ce temps est de l'ordre de 2 minutes : ici, le potentiel s'établit en 70 à 90 secondes, et lors de l'addition d'un cation divalent, en 6 secondes seulement ; l'équation de Gouv et Chapman est, en première approximation, applicable, et nous sommes en présence de potentiels d'interface créés par l'addition de $\mathrm{Mg}^{++}$;

(2) Le magnésium agit sur la différence de potentiel et sur la résistance des membranes artificielles lipidiques:

- il agit par effet d'écran, comme $\mathrm{Ca}^{++}$et $\mathrm{Sr}^{++}$(potentiel inférieur à $27 \mathrm{mV}$ ), alors que $\mathrm{Ni}^{++}, \mathrm{Ba}^{++}, \mathrm{Mn}^{++}$se fixent) ; c'est-à-dire qu'il y a neutralisation des charges négatives et diminution de la conductance; en fait, nous trouvons ces résultats pour les membranes de lécithine et de céphaline, mais pour des membranes de lipides totaux, nous trouvons:

- à faibles concentrations, $\mathrm{Mg}^{++}$a peu d'effet, mais dans le sens prévu ;

-- à fortes concentrations, la conductance augmente; on peut alors envisager :

- soit un déséquilibre du champ électrique, peu probable, car les variations de potentiel sont peu importantes ;

- soit une augmentation du nombre de charges positives et une augmentation de la conductance au chlore; ceci n'étant pas valable pour $\mathbf{M g}^{++}$, cation divalent le plus gros (rayon de $\mathrm{Mg}^{++}$hydraté $=5,9 \AA$ ) ;

- soit une déformation du réseau par modification d'orientation des dipôles et du potentiel dipolaire.

Ces résultats tendent à montrer que $\mathbf{M g}^{++}$, ainsi que les autres cations divalents, est adsorbé spécifiquement sur les groupes polaires; ce point est confirmé par la compétition entre $\mathrm{Mg}^{++}$et les cations monovalents : en effet, si on trace la courbe $\mathrm{I} / \mathrm{g}=\mathrm{f}\left(\mathrm{Mg}^{++}\right)$(fig. 4) (représentation des cinétiques enzymatiques de Dixon), pour une solution de $\mathrm{KCl}$ par exemple, on voit que $\mathrm{I} / \mathrm{g}$ varie de façon linéaire en fonction de l'augmentation de la concentration en ion $\mathrm{Mg}^{++}$: on peut dire que $\mathrm{Mg}^{++}$est compétitif des cations monovalents au niveau des mêmes sites sur le phospholipides.

\section{CONCLUSION}

L'ion magnésium agit sur les membranes artificielles liquides et bimoléculaires lipidiques, par effet d'écran, en augmentant la résistance des membranes à charge résultante positive et en diminuant celle des membranes à charge résultante négative ; il crée aussi un potentiel membranaire notable. Il agit aussi, comme compétiteur des ions monovalents, surtout de l'ion potassium au niveau des mêmes groupes polaires des phospholipides. 


\title{
SUMMARY
}

\author{
EFFECTS OF MAGNESIUM ON LIQUID \\ AND PHOSPHOLIPIDIC BIMOLECULAR MEMBRANES. \\ THE MAGNESIUM ION, A MONOVALENT CATION COMPETITOR
}

Little is known about the structure of cell membranes. They are composed of lipidic and proteinic units, and some physical parameters (the nature of the ionic species, for example) act on lipidic molecule orientation and on proteins. Using artificial membranes of a "liquid " type (phospholipids dissolved in an organic solvent having low dielectric constant and impregnating a millipore teflon filter), or phospholipidic bimolecular membranes, we try to show the role of divalent cations, and particularly that of $\mathrm{Mg}^{++}$. Resistance and membrane potential are measured using a Keithley $6 \mathrm{IoB}$ electrometer.

Magnesium and other divalent cations act on the structural stability of the membrane. This action is expressed by an increased resistance of membranes having positive resultant charge (cephalin and lecithin), and a drop in resistance of those having negative resultant charge (total lipids of the beef brain). Moreover, the difference of membrane potential is affected (more clearly with total lipids).

$\mathrm{Mg}^{++}$action is explained by applying the double layer theory of Gouy and CHapman. $\mathrm{Mg}^{++}$has a screening effect ; it is adsorbed specifically on polar groups of the membrane. This is confirmed by curves, $\mathrm{I} / \mathrm{g}=\mathrm{f}\left(\mathrm{Mg}^{++}\right)$, which show that $\mathrm{Mg}^{++}$is a monovalent cation competitor in the same sites of the membrane.

\section{RÉFÉRENCES BIBLIOGRAPHIQUES}

Benson A. A., I966. On the orientation of lipids in chloroplast and cell membranes. J. Am. Oil. Soc., 43, 265-270.

Danielli J. F., Davson H., 1935. A contribution of the theory of permeability of thin lipids. J. Cell. Comp. Physiol., 5, 495-508.

Gilbert D. L., Ehrenstein G., I969. Effect of divalents cations on potassium conductance of squid axons : determination of surface charge. Biophys. J., 9, 447-464.

Gorter E., Grendel F., I925. Bimolecular layers of lipoids on chromocytes of blood. J. Exptl. Med., 41, 439-443.

Green D. E., Perdue J. F., 1966. Membranes as expressions of repeating units. Proc. Natl. Acad. Sci. U.S., 55, 1295-I302.

Hodgkin A. L., Huxley A. F., 1952. A quantitative description of membrane current and its application to conduction and excitation in nerve. J. Physiol. (London), 117, 500-544.

Krasne S., Eisenman G., Szabo G., I97I. Freezing and melting of lipid bilayers and the mode of action of nonactin, valinomycin and gramicidin. Science, 174, 4I2-4I5.

Lenard J., Singer S. J., I966. Protein conformation in cell membrane preparation as studied by optical rotatory dispersion and circular dichroïsm. Proc. Natl. Acad. Sci. U. S., 56, r828-I835.

Lucy J. A., 1964. Globular lipid micelles and cell membranes. J. Theoret. Biol., 7, 360-368.

Me Donald R. C., Bangham A. D., r972. Comparison of double layer potential of charged phospholipid membranes. J. Memb. Biol., 7, 29-53.

Mc Laughlin S. G. A., Szabo G., Eisenman G., I97I. Divalents ions and the surface potential of charged phospholipid membranes. J. Gen. Physiol., 58, 667-687.

Mueller P., Rudin D. O., Tien H. T., Wescott W. C., I962. Reconstitution of cell membrane structure in vitro and its transformation into an excitable system. Nature, 184, 979-980.

Stein W. D., Danielli J. F., 1956. Structure and fuction in red cell permeability. Dis. Farad. Soc., 21, 238-252. 